\title{
Recent Developments in Glaucoma
}

\author{
An Expert Interview with Kin Sheng Lim
}

Guy's and St Thomas' Hospitals, London, UK

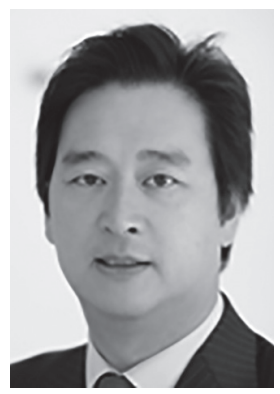

DOI: https://doi.org/10.17925/EOR.2017.11.02.87

\begin{abstract}
Kin Sheng Lim
Mr Kin Sheng Lim qualified from University of Leicester Medical School and undertook his MD research at University College, London. His general ophthalmology training and doctorate research into glaucoma surgery took place in London and Brighton. He also spent a year completing a post-doctoral fellowship at the Mayo Clinic in Rochester, MN, US, supported by a scholarship from the International Glaucoma Association. For over 10 years, Mr Sheng Lim has headed the Glaucoma Service at St Thomas' Hospital where he is also currently head of research in the ophthalmology department. His clinical interests are in the treatment of glaucoma and high-volume cataract surgery. He supervises a research team of 10 clinical and support staff and is currently conducting 14 clinical trials into the pathogenesis and new treatment options for glaucoma.
\end{abstract}

\section{Keywords}

Glaucoma, intraocular pressure, IOP, rho kinase inhibitors, minimally invasive glaucoma surgey, MIGS

Disclosure: Kin Sheng Lim has consultancy agreements with Alcon, Allergan, Santen and Ivantis, and currently receives research funding from New World Medical, Iridex, Thea, Alcon and EyeTechCare to declare in relation to this article. This is an expert interview and as such, has not undergone the journal's standard peer review process.

Acknowledgments: Medical writing assistance was provided by James Gilbart from Touch Medical Media.

Authorship: All named authors meet the International Committee of Medical Journal Editors (ICMJE) criteria for authorship of this manuscript, take responsibility for the integrity of the work as a whole, and have given final approval to the version to be published.

Open Access: This article is published under the Creative Commons Attribution Noncommercial License, which permits any non-commercial use, distribution, adaptation and reproduction provided the original author(s) and source are given appropriate credit.

Received: 14 December 2017

Published Online: 22 December 2017

Citation: European Ophthalmic Review, 2017;11(2):87-9

Corresponding Author: Kin Sheng Lim, KCL Frost Eye Research Department, St Thomas' Hospital, Westminster Bridge Road, London SE1 7EH, UK. E: shenglim@gmail.com

Support: This expert interview was not supported by any external funding.
G laucoma continues to exert a heavy disability and economic burden and is the most frequent cause of irreversible blindness worldwide. ${ }^{1}$ In 2015, 57.5 million people globally were affected by open-angle glaucoma and this is predicted to increase to 65.5 million by 2020. ${ }^{2}$ The prevalence of glaucoma increases with age in all populations and generally more men and people of Hispanic and Black race are affected. Effective treatments to lower and maintain intraocular pressure (IOP) and convenient means of continuously monitoring it remain substantial unmet medical needs. In this expert interview Kin Sheng Lim from Guy's and St Thomas' Hospitals, London, UK, discusses the new developments to improve this situation and are therefore of great interest to ophthalmologists and to the wider population who are at risk from glaucoma.

\section{Q: What have been the major developments in pharmacotherapy for glaucoma in 2017?}

One of the most exciting developments in glaucoma pharmacotherapy over the past 12 months has been the progress in the development of rho kinase inhibitors. The mechanism of action of these treatments has not been fully elucidated but it is entirely different to those of existing medications for glaucoma such as prostaglandins and beta-blockers. Rho kinases are serine/ threonine kinases that have multiple functions within various different cell types throughout the body and are key components in regulating cell shape, motility, proliferation and apoptosis. ${ }^{3-5}$ Rho kinases achieve these effects by regulating smooth muscle contraction, which results from their interaction with multiple actin cytoskeletal-related targets. Various studies have shown that loss of rho kinase activity is associated with micromechanical relaxation of cells and disassembly of stress fibres and focal adhesion complexes. ${ }^{6.7}$ In the eye, rho kinase increases the contractile properties of trabecular meshwork (TM) outflow tissue. Inhibition of rho kinase activity therefore allows increased aqueous outflow via the TM and decreased intraocular pressure. ${ }^{5}$

A variety of different rho kinase inhibitors are in development for use in glaucoma of which, ripasudil and netarsudil are at the most advanced stage. ${ }^{5}$ In a one-year open-label study $(n=354)$, ripasudil also produced notable reductions in IOP when given as monotherapy, as adjunctive therapy with prostaglandins, beta-blockers or with fixed combination drugs, and was well tolerated. ${ }^{8}$ In two phase III studies $(n=1,167)$, treatment with netarsudil $0.2 \%$ once daily, produced significant reductions from baseline IOP $(p<0.001)$ and was non-inferior to timolol twice daily. ${ }^{9}$ This treatment was well tolerated, with transient conjunctival hyperaemia being the most frequent adverse event. Based on this evidence, the US Food and Drug Administration (FDA) has recently approved Rhopressa ${ }^{\circledast}$ (netarsudil) for the treatment of glaucoma in the US. ${ }^{10}$ 
A further exciting development is the appearance of slow-release formulations and methods for less frequent dosing of prostaglandins. Examples include ENV515, which is travoprost in a biodegradable polymer for intracameral injection. ${ }^{11}$ A recent phase II study showed that one injected dose of ENV515 maintained an IOP reduction of 26\% for up to 9 months with no serious adverse events or corneal effects. Another development is an ocular ring insert containing bimatoprost, which is placed on the eye to release the drug over extended durations. Its efficacy was demonstrated in a phase II study in which the insert produced similar IOP-lowering performance over 6 months to that of a placebo insert and twice daily $0.5 \%$ timolol twice daily, and was well tolerated. ${ }^{12}$

The iDose ${ }^{\circledast}$ (Glaukos, San Clemente, CA, US) delivery system is another device that is being evaluated in glaucoma. This is an intraocular implant that is injected during a micro-invasive procedure after which it elutes $0.5 \%$ travoprost over extended periods. The iDose is currently being compared with topical timolol maleate $0.5 \%$ in the treatment of glaucoma in a randomized double-blind, phase $\|$ trial $(n=154) .{ }^{13}$ Recruitment was completed in May 2017. The trial was expected to conclude by the end 2017 and phase III trials will commence in early 2018. A bimatoprost slow-release (SR) formulation (Allergan, Dublin, Ireland) for intracameral administration is also in development for glaucoma. A recent phase I/ II study ( $n=75$ ) compared $6,10,15$ or $20 \mu$ g versions of this SR implant in one eye of each patient with topical bitamoprost $0.03 \%$ in the fellow eye over a 24-month period. Six-month results show that bimatoprost-SR reduced IOP by $7.2,7.4,8.1$ and $9.5 \mathrm{mmHg}$, respectively, compared with $8.4 \mathrm{mmHg}$ for topical bimatoprost. ${ }^{14,15}$ Conjunctival hyperaemia within 2 days of administration was the most common adverse event occurring in $6.7 \%$ of SR-treated eyes and $17.3 \%$ of topically treated eyes. Overall, the SR treatment showed favourable efficacy over 6 months.

In addition to reducing treatment burden, these new implantable SR and wearable devices have the potential to improve patient adherence and enable better continuous control of IOP.

\section{Q: How has our understanding of glaucoma advanced in 2017 ?}

A recent change in perception concerns the practice or iridotomy for glaucoma treatment. In 2014, a study by Vera et al. ( $n=208),{ }^{16}$ showed that temporal placement of laser peripheral iridotomy was safe and less likely to result in linear dysphotopsia than superior placement (10.7\% versus 2.4\%). This finding influenced many ophthalmic surgeons to switch from superior to temporal placement. More recently, however, a larger study by Srinivasan et al. in India $(n=559)^{17}$ showed that dysphotopsia incidence was similar regardless of iridotomy location (8.4\% for superior versus $9.5 \%$ for temporal; $p=0.7$ ). This finding may persuade ophthalmic surgeons to revert back to using superior iridotomy for glaucoma treatment, as was their previous practice.

A further interesting development comes from the Primary Tube versus Trabeculectomy (PTVT) study ( $n=117) .{ }^{18-20}$ One-year results showed that for patients with uncontrolled glaucoma, who did not have any previous intraocular surgery, trabeculectomy with Mitomycin-C had a higher success rate and lower IOPS compared with those receiving tube shunt placement. Furthermore, the greater IOP reduction was achieved with fewer glaucoma medications ( $p<0.001$ at 1 week, 1 month, 3 months, 6 months and 1 year for both IOP and medications). In this study, there were no significant differences in the rates of intraoperative complications, late postoperative complications or serious complications between the two groups, and most were transient and self limited.
Other changes in understanding come from the EAGLE study $(n=419) .{ }^{21}$ Although the study findings were published in 2016, its implications are only now becoming fully appreciated. The results showed that in patients with primary angle closure glaucoma, clear-lens extraction produced greater reductions in IOP $(p=0.004)$, higher scores for quality of life measures $(p=0.005)$ and was more cost effective than laser peripheral iridotomy (incremental cost effectiveness ratio was $£ 14,284$ for initial lens extraction versus standard care). Based on this, the authors suggested that clear-lens extraction should be considered as an option for first-line treatment for better outcome and cost effectiveness in this subgroup of patients.

For many ophthalmologists the central 24-2 threshold test has become the standard method for investigating visual field defects in glaucoma. However, in a recent study in the US ${ }^{22}$ in patients with suspected glaucoma, 79 of the 200 eyes (39.5\%) classified as normal on the $24-2$ test were classified as abnormal on 10-2 visual fields. In ocular hypertensive eyes, 28 of the 79 eyes (35.4\%) classified as normal on the 24-2 test were classified as abnormal on the 10-2 test. These findings suggest that it may be necessary to switch from the 24-2 to the 10-2 test in the next few years to more reliably detect visual defects in some patients.

\section{Q: What advances have there been in minimally invasive glaucoma surgery (MIGS) in 2017 and which approaches appear most promising?}

The recent FDA approval of the CyPass ${ }^{\circledast}$ trabecular micro-stent (Alcon/ Novartis, Fort Worth, TX, US) is a key development that is likely to change practice in glaucoma surgery. ${ }^{23}$ This was supported by the 2-year results from the pivotal COMPASS trial on patients $(n=505)$ with unmedicated IOPS in the range $21-33 \mathrm{mmHg}$ and receiving pharmacoemulsification cataract surgery. ${ }^{24}$ Study findings showed an early and sustained reduction in IOP in $77 \%$ of microstent subjects achieving $\geq 20 \%$ unmedicated IOP lowering versus baseline at 24 months compared with $60 \%$ of controls ( $p=0.001$ ). In addition, mean 24-month medication was $67 \%$ lower in patients receiving CyPass $(p<0.001)$ and there were no CyPass-related adverse events. No vision-threatening CyPass-related adverse events occurred; long-term safety data from the COMPASS trial will become available during 2018. The availability of CyPass may therefore encourage greater adoption of MIGS techniques for glaucoma treatment.

A further development in MIGS is the Hydrus ${ }^{\mathrm{TM}}$ Schlemm canal microstent (Ivantis, Dublin, Ireland) for which FDA licensing application for use in glaucoma is ongoing. In the HORIZON study 556 patients with cataract and mild to moderate glaucoma were randomly assigned to cataract surgery with the Hydrus or to cataract surgery alone..25 Two-year results show that $77.2 \%$ of patients who received the Hydrus microstent achieved a $20 \%$ reduction in IOP versus $57.8 \%$ who received cataract surgery alone. The SUMMIT trial has been recently approved by the FDA and will evaluate Hydrus Microstent in refractory glaucoma which will add to the data gained from mild-to-moderate glaucoma cases in previous studies. ${ }^{26}$

These new devices may have great potential in glaucoma treatment, but they are limited by a lack of head-to-head studies comparing their performance. It will take some years before their relative merits are fully appreciated and those that provide optimal patient outcomes emerge.

\section{Q: What other emerging technologies have interested you this year?}

One other emerging technology is the Microshunt ${ }^{\circledast}$ (Innfocus, Miami, FL, US). ${ }^{27}$ This device is the first 'minimally invasive' standalone procedure introduced in recent years for open angle glaucoma of any stage or severity. Data from a 3-year long-term study showed that after the 
MicroShunt procedure, mean IOP was reduced by $55 \%$ to $10.7 \mathrm{mmHg}$ and more than $80 \%$ of the 22 patients had an IOP below $14 \mathrm{mmHg} .{ }^{28}$ In addition, $64 \%$ of patients did not require any glaucoma medication during the third year of the study. A phase I//II study with a planned population of 857 patients is currently ongoing (NCT01881425). This will compare the safety and efficacy of MicroShunt to standard trabeculectomy in subjects with primary open angle glaucoma and the data are intended to support an FDA filing for use of the device in glaucoma.

\section{Q: What future developments can we look forward to in 2018 ?}

In most patients with glaucoma, IOP monitoring is usually conducted irregularly at clinic visits and consequently cannot capture diurnal variations and spikes that can be damaging to the eye and need to be eliminated through increased treatment and/or altered dosing regimens. ${ }^{29}$ Various invasive and non-invasive systems have been investigated to continuously monitor IOP but to date none have proven entirely satisfactory. These systems are of three main types: self-monitoring, temporary continuous monitoring and permanent continuous monitoring. ${ }^{30}$ An example of a wearable device is the Triggerfish ${ }^{\circledR}$ contact lens sensor (SENSIMED, Lausanne, Switzerland). This device can only measure relative changes rather than determine absolute pressure. ${ }^{31}$ There are currently many new intraocular sensor devices in development ${ }^{32-34}$ and if any of these are proven to be accurate and safe, reliable continuous IOP monitoring for many patients may soon become a reality. $\square$
1. Pascolini $D$, Mariotti $S P$, Global estimates of visual impairment: 2010, Br J Ophthalmol, 2012;96:614-8.

2. Kapetanakis WV, Chan MP, Foster PJ, et al., Global variations and time trends in the prevalence of primary open angle glaucoma (POAG): a systematic review and meta-analysis, Br J Ophthalmol, 2016;100:86-93.

3. Rao VP, Epstein DL, Rho GTPase/Rho kinase inhibition as a novel target for the treatment of glaucoma, BioDrugs, 2007;21:167-77.

Riento K, Ridley AJ, Rocks: multifunctional kinases in cell

behaviour, Nat Rev Mol Cell Biol, 2003;4:446-56.

5. Wang SK, Chang RT, An emerging treatment option for glaucoma: Rho kinase inhibitors, Clin Ophthalmol, 2014;8:883-90.

6. Ishizaki T, Maekawa M, Fujisawa K, et al., The small GTP-binding protein Rho binds to and activates a $160 \mathrm{kDa}$ Ser/Thr protein kinase homologous to myotonic dystrophy kinase, EMBO J, 1996; 15:1885-93

7. Uehata M, Ishizaki T, Satoh $\mathrm{H}$, et al., Calcium sensitization of smooth muscle mediated by a Rho-associated protein kinase in hypertension, Nature, 1997;389:990-4.

8. Tanihara $\mathrm{H}$, Inoue $\mathrm{T}$, Yamamoto $\mathrm{T}$, et al., One-year clinical evaluation of $0.4 \%$ ripasudil ( $\mathrm{K}-115)$ in patients with openangle glaucoma and ocular hypertension, Acta Ophthalmol 2016;94:e26-34

9. Serle JB, Katz $\sqcup$, McLaurin $E$, et al., Two phase 3 clinical trials comparing the safety and efficacy of netarsudil to timolol in patients with elevetedintracular pressure, Am J Ophthalmo, 2017; doi: 10.1016/j.ajo.2017.11.019 [Epub ahead of print].

10. Aerie Pharmaceuticals, Aerie Pharmaceuticals Announces US FDA Approval of Rhopressa® (netarsudil ophthalmic solution) $0.02 \%$ for the Lowering of Elevated Intraocular Pressure in Patients with Open-Angle Glaucoma or Ocular Hypertension. Available at: www.drugs.com/nda/rhopressa_171013.htm (accessed 15 January 2018)

11. Navratil T, Garcia A, Tully J, Preclinical evaluation of ENV515 (travoprost) intracemeral implant-clinical candidate for treatment of glaucoma targeting six-month duration of action, Presented at: Association for Research in Vision and Ophthalmology (ARVO), Orlando, FL, US, 4-8 May 2014

12. Brandt JD, Sall K, DuBiner $H$, et al., Six-month intraocular pressure reduction with a topical bimatoprost ocular insert: Results of a phase II randomized controlled study, Ophthalmology, 2016;123:1685-94.

13. Glaukos Press release: Glaukos Completes Patient Enrollment in Phase II Clinical Trial for iDose TM Travoprost Intraocular Implant in Glaucoma Patients, 2017. Available at: http://investors.glaukos. com/investors/press-releases/press-release-details/2017/ Glaukos-Completes-Patient-Enrollment-in-Phase-II-Clinical-Trialfor-iDose-Travoprost-Intraocular-Implant-in-Glaucoma-Patients/ default.aspx (accessed 11 December 2017).

14. Allergan - News, Positive Phase I/II Interim Data of Bimatoprost Sustained-Release Implant for IOP Therapy in Glaucoma Phase I/II Interim Data Show Implant was Tolerated and is Comparable to Daily Topical Bimatoprost in Efficacy, 2015. Available at: www.allergan.com/news/news/thomson-reuters/ positive-phase-i-ii-interim-data-of-bimatoprost-su (accessed 11 December 2017)

15. Lewis RA, Christie WC, Day DG, et al., Bimatoprost sustainedrelease implants for glaucoma therapy: 6-month results from a phase I/II clinical trial, Am J Ophthalmol, 2017;175:137-47.

16. Vera V, Naqi A, Belovay GW, et al., Dysphotopsia after temporal versus superior laser peripheral iridotomy: a prospective randomized paired eye trial, Am J Ophthalmol, 2014;157:929-35.

17. Srinivasan K, Zebardast N, Krishnamurthy P, et al., Comparison of new visual disturbances after superior versus nasal/temporal laser peripheral iridotomy: A prospective randomized trial, Ophthalmology ، 2017; doi: 10.1016/.ophtha.2017.09.015 [Epub ahead of print].

18. Healio Ocular Surgery, Trabeculectomy more successful than tube shunt in Primary TVT study, 2016. Available at: wnw.healio. com/ophthalmology/glaucoma/news/online/\%7B06c356e0-797745c1-9819-9531639d1d4e\%7D/trabeculectomy-more-successfulthan-tube-shunt-in-primary-tvt-study (accessed 07 December 2017)

19. Gedde S, Treatment outcomes in the Primary Tube Versus Trabeculectomy (PTVT) study after 1 year of follow-up. Presented at: American Academy of Ophthalmology annual meeting Chicago, Il, Oct. 14-18, 2016, 2016,

20. Lim S, Postoperative complications in the Primary Tube Versus Trabeculectomy (PTVT) study during the first year of follow-up, Presented at: American Academy of Ophthalmology Annual Meeting, Chicago, II, US, 14-18 October 2016.

21. Azuara-Blanco A, Burr J, Ramsay C, et al., Effectiveness of early lens extraction for the treatment of primary angle-closure glaucoma (EAGLE): a randomised controlled trial, Lancet, 2016;388:1389-97.

22. De Moraes $\mathrm{CG}$, Hood DC, Thenappan A, et al., 24-2 Visual Fields Miss Central Defects Shown on 10-2 Tests in Glaucoma Suspects, Ocular Hypertensives, and Early Glaucoma, Ophthalmology, 2017;124:1449-56.

23. Healio Ocular Surgery News, FDA approves CyPass microstent system for use with cataract surgery, 2016. Available at: www.healio.com/ophthalmology/cataract-surgery/news/
online/\%7Bde7371c2-09a1-46de-96f0-94c25736b7b2\%7D/ fda-approves-cypass-micro-stent-system-for-use-with-cataractsurgery (accessed 07 December 2017).

24. Vold S, Ahmed, II, Craven ER, et al., Two-Year COMPASS Trial Results: Supraciliary Microstenting with Phacoemulsification in Patients with Open-Angle Glaucoma and Cataracts, Ophthalmology, 2016;123:2103-12

25. Helio Ocular Surgery News, Hydrus canal stent meets study endpoint, 2017. Available at: www.healio.com/ophthalmology/ glaucoma/news/online/\%7B2205ec23-79e2-4ab1-baec$108 \mathrm{e} 0337 \mathrm{~d} 23 \%$ \% 7 /hydrus-canal-stent-meets-study-endpoint (accessed 07 December 2017).

26. Marketwired, Ivantis Announces FDA Clearance to Initiate Clinical Trial of the Hydrus ${ }^{\top M}$ Microstent for Minimally Invasive Glaucoma Surgery (MIGS) in Patients with Advanced Glaucom 2017. Available at: www.marketwired.com/press-release/ ivantis-announces-fda-clearance-initiate-clinical-trial-hydrusmicrostent-minimally-2211676. html (accessed 07 December 2017)

27. Cision PR Newswire, InnFocus MicroShunt $\circledast 3$-Year Results of a Long Term Clinical Trial Show Long Lasting IOP Reduction, 2016. Available at: www.prnewswire.com/news-releases/ innfocus-microshunt-3-year-results-of-a-long-term-clinical-trialshow-long-lasting-iop-reduction-300210457.html (accessed 07 December 2017).

28. Batlle JF, Fantes F, Riss I, et al., Three-Year Follow-up of a Nove Aqueous Humor MicroShunt, I Glaucoma, 2016;25:e58-65.

29. MCMonnies $\mathrm{CW}_{\text {, The importance of and potential for }}$ continuous monitoring of intraocular pressure, Clin Exp Optom, 2017;100:203-7.

30. Ittoop SM, SOOHoo JR, Seibold LK, et al., Systematic Review of Current Devices for 24-h Intraocular Pressure Monitoring, $A d v$ Ther, 2016;33:1679-90

31. Dunbar GE, Shen BY, Aref AA, The Sensimed Triggerfish contact Iens sensor: efficacy, safety, and patient perspectives, Clin Ophthalmol, 2017;11:875-82.

32. Bello $\mathrm{SA}$, Passaglia $\mathrm{CL}$, A Wireless Pressure Sensor for Continuous Monitoring of Intraocular Pressure in Conscious Animals, Ann Biomed Eng, 2017; 45:2592-604.

33. Kim YW, Kim MJ, Park KH, et al., Preliminary study on implantable inductive-type sensor for continuous monitoring of intraocular pressure, Clin Exp Ophthalmol, 2015;43:830-7.

34. Mariacher $\mathrm{S}$, Ebner $\mathrm{M}$, Januschowski $\mathrm{K}$, et al., Investigation of a novel implantable suprachoroidal pressure transducer of a novel Implantable suprachoroidal pressure transducer 2016;151:54-60. 